\title{
Phylogeography of Francisella tularensis subspecies holarctica from the country of Georgia
}

\author{
Gvantsa Chanturia $^{2 \dagger}$, Dawn N Birdsell ${ }^{1 \dagger}$, Merab Kekelidze ${ }^{2}$, Ekaterine Zhgenti ${ }^{2}$, George Babuadze $^{2}$, \\ Nikoloz Tsertsvadze², Shota Tsanava², Paata Imnadze², Stephen M Beckstrom-Sternberg ${ }^{3}$, \\ James S Beckstrom-Sternberg ${ }^{3}$, Mia D Champion ${ }^{3}$, Shripad Sinari ${ }^{3}$, Miklos Gyuranecz ${ }^{4}$, Jason Farlow ${ }^{5}$, \\ Amanda H Pettus ${ }^{1}$, Emily L Kaufman ${ }^{1}$, Joseph D Busch ${ }^{1}$, Talima Pearson ${ }^{1}$, Jeffrey T Foster ${ }^{1}$, Amy J Vogler ${ }^{1}$, \\ David M Wagner ${ }^{1}$ and Paul Keim ${ }^{*}$
}

\begin{abstract}
Background: Francisella tularensis, the causative agent of tularemia, displays subspecies-specific differences in virulence, geographic distribution, and genetic diversity. F. tularensis subsp. holarctica is widely distributed throughout the Northern Hemisphere. In Europe, F. tularensis subsp. holarctica isolates have largely been assigned to two phylogenetic groups that have specific geographic distributions. Most isolates from Western Europe are assigned to the B.Br.FTNF002-00 group, whereas most isolates from Eastern Europe are assigned to numerous lineages within the B.Br.013 group. The eastern geographic extent of the B.Br.013 group is currently unknown due to a lack of phylogenetic knowledge about populations at the European/Asian juncture and in Asia. In this study, we address this knowledge gap by describing the phylogenetic structure of $F$. tularensis subsp. holarctica isolates from the country of Georgia, and by placing these isolates into a global phylogeographic context.
\end{abstract}

Results: We identified a new genetic lineage of F. tularensis subsp. holarctica from Georgia that belongs to the B.Br.013 group. This new lineage is genetically and geographically distinct from lineages previously described from the B.Br.013 group from Central-Eastern Europe. Importantly, this new lineage is basal within the B.Br.013 group, indicating the Georgian lineage diverged before the diversification of the other known B.Br.013 lineages. Although two isolates from the Georgian lineage were collected nearby in the Ukrainian region of Crimea, all other global isolates assigned to this lineage were collected in Georgia. This restricted geographic distribution, as well as the high levels of genetic diversity within the lineage, is consistent with a relatively older origin and localized differentiation.

Conclusions: We identified a new lineage of F. tularensis subsp. holarctica from Georgia that appears to have an older origin than any other diversified lineages previously described from the B.Br.013 group. This finding suggests that additional phylogenetic studies of F. tularensis subsp. holarctica populations in Eastern Europe and Asia have the potential to yield important new insights into the evolutionary history and phylogeography of this broadly dispersed F. tularensis subspecies.

\section{Background}

Francisella tularensis is a highly clonal, recentlyemerged pathogen that causes tularemia, which presents in several main forms: pneumonic (30\%-60\% mortality), ulceroglandular, and oropharyngeal [1]. The latter two are associated with lower mortality. F. tularensis is

\footnotetext{
* Correspondence: Paul.Keim@nau.edu

+ Contributed equally

${ }^{1}$ Center for Microbial Genetics and Genomics, Northern Arizona University, Flagstaff, AZ 86011-4073, USA

Full list of author information is available at the end of the article
}

currently divided into three subspecies (tularensis, holarctica and mediasiatica), with F. novicida recognized as a very closely related species, or as another subspecies by some authors [2-4]. These taxa vary in virulence, geographic distribution, overall genetic diversity, and host/vector associations [3,5-9]. Human tularemia is a disease at which the clinical severity depends upon the route of infection, subspecies of the infection strain, and timely therapeutic response [9]. Cases in Europe are caused by $F$. tularensis subsp. holarctica, and in
Ciomed Central

() 2011 Chanturia et al; licensee BioMed Central Ltd. This is an Open Access article distributed under the terms of the Creative Commons Attribution License (http://creativecommons.org/licenses/by/2.0), which permits unrestricted use, distribution, and reproduction in any medium, provided the original work is properly cited. 
many rural areas of the Balkans and countries further east outbreaks are water-borne, resulting in oropharyngeal tularemia [10-12]. No known cases by F. tularensis subsp. mediasiatica are known and only a few by $F$. novicida have been documented $[13,14]$. F. tularensis subsp. tularensis is restricted to North America, whereas F. tularensis subsp. holarctica is found throughout the Northern Hemisphere [3,15]. Despite its wider geographic distribution $F$. tularensis subsp. holarctica has markedly lower genetic diversity than F. tularensis subsp. tularensis $[5,7,8]$.

Significant gains toward deciphering the evolutionary history of $F$. tularensis overall and, in particular, F. tularensis subsp. holarctica have been made by using whole genome comparisons for single nucleotide polymorphism (SNP) discovery coupled with subsequent canonical SNP (canSNP) analysis $[15,16]$. Numerous new groups were identified within $F$. tularensis subsp. holarctica (Figure 1A) $[15,16]$, two of which, B.Br.013 (includes subclades B. Br.013/014 and B.Br.LVS in [15]) and B.Br.FTNF002-00, were predominant in Europe but geographically segregated [15]. In the Western European countries of Spain, France, and Switzerland almost all isolates belong to the highly monomorphic B.Br.FTNF002-00 group [15-18]. In contrast, in large portions of Central and Eastern Europe, from the Czech Republic to Russia, most F. tularensis subsp. holarctica isolates are assigned to various lineages within the B.Br.013 group $[15,16]$.

Additional analyses of the B.Br.013 group are crucial for fully understanding the phylogeography of $F$. tularensis subsp. holarctica in Europe and Asia. This group contains significant genetic diversity based upon multilocus variable-number tandem repeat (VNTR) analysis (MLVA) [15], indicating that considerable phylogenetic structure may exist that could be revealed with additional analyses. In addition, this group is widely distributed, extending from Eastern Europe into the border regions of the European/Asian continents. Importantly, the eastern geographic extent of the B.Br.013 group is very poorly understood. This is because, to date, it has not been possible to place $F$. tularensis isolates from countries at the boundary of the European/Asian continents and Western Asia, including Georgia, into a larger phylogeographic context. Based on growth characteristics, biochemical analyses, basic PCR methods, and DNA sequencing, we know that $F$. tularensis subsp. holarctica is the predominant subspecies in Georgia and in regions further east [11,19-21], but more specific genetic information is limited. Some isolates from the European/Asian juncture regions and East Asia have been genotyped with a subset of VNTRs but have not been part of any global analyses [10,22,23]. Although valuable for regional studies, homoplasy associated with these rapidly-evolving markers restricts their value for global phylogenetic analyses [24].

In this study, we determined the phylogenetic structure of F. tularensis subsp. holarctica isolates from the European/Asian juncture country of Georgia by sequencing the genome of a Georgian isolate, comparing that genome to other available whole genome sequences to discover SNPs, and screening a subset of the resulting SNPs across 25 isolates from Georgia. We examined diversity within the subclades defined by these SNPs using a multiple-locus variable number tandem repeat analysis (MLVA) system [25]. To place the Georgian isolates into an existing global phylogeographic framework [15], we also screened a canonical subset of the newly discovered SNPs across a large panel of European isolates belonging to the B.Br.013 group.

\section{Results}

\section{Georgian isolate whole genome sequence}

Initial analyses with previously described canSNP assays (See Additional file 1, [15]) revealed that all 25 Georgian isolates belong to the B.Br.013 group. One of the Georgian strains (F0673) was sequenced using the Illumina Genome Analyzer II sequencing platform resulting in very high sequence coverage (averaging $1,076 \mathrm{X}$ ) when aligned to the LVS genome (See Additional file 2, [26]). Subsequent whole genome sequence (WGS) comparisons among three published B.Br.013 group genomes (FSC 200, LVS, and RC503), the genome of strain F0673 generated for this study, and the published OSU18 genome (as an outgroup) revealed 650 putative SNPs. Most of these putative SNPs ( $\mathrm{n}=$ 470) were phylogenetically located on the branches separating OSU18 from the genomes in the B.Br.013 group (data not shown). Maximum parsimony analysis of the putative SNPs produced a phylogeny (Figure 1B) with a very low homoplasy index (0.02), consistent with the highly clonal nature of $F$. tularensis. The phylogenetic topology of the FSC 200, LVS, and RC503 genomes is consistent with previous publications $[15,16]$, and the small number of putative SNPs unique to the Georgian strain is consistent with the low genetic diversity observed among other lineages within F. tularensis subsp. holarctica $[3,6,27,28]$. The new branch (B.Br.027) leading to the Georgian strain arises from a common ancestor that is basal to the previously described diversified lineages within the B.Br.013 group and is separated from them by only 45 putative SNPs, with 39 of these putative SNPs leading to the Georgian strain (B.Br.027 in Figure 1B) and the other six putative SNPs along a branch (B.Br.026 in Figure 1B) defining a monophyletic lineage containing the other sequenced strains from this group. 

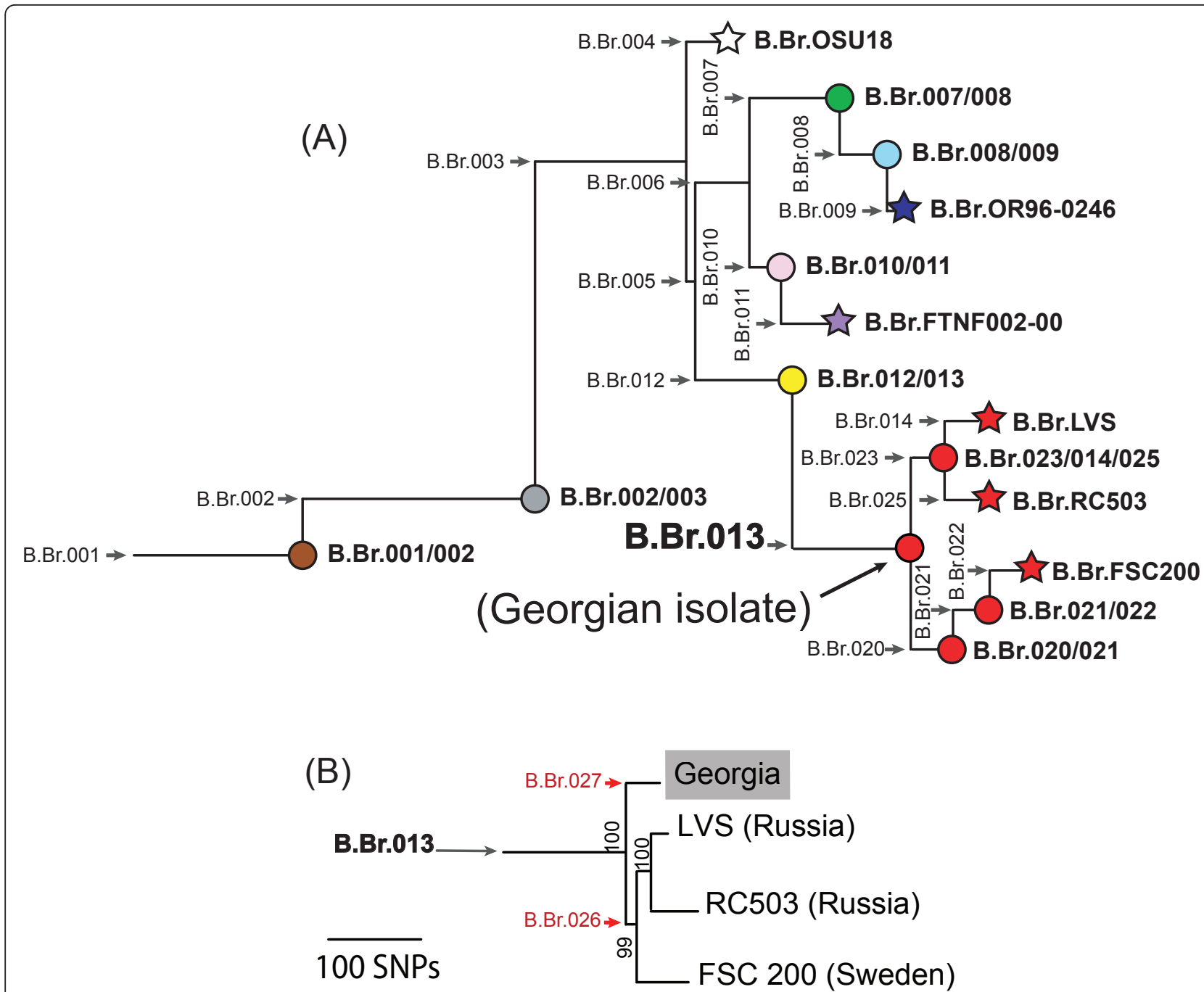

Figure 1 Phylogenies of Francisella tularensis subsp. holarctica. (A) CanSNP phylogeny of Francisella tularensis subsp. holarctica subclades identified by Vogler et al. and Svensson et al. $[15,16]$ (See additional file 1 for an update of these SNP positions based on the latest SCHU S4 genome NC_006570). Subclades within the B.Br.013 group are depicted in red. The Georgian isolate was placed in the basal node B.Br.013/020/ 023 (black arrow). (B) Maximum parsimony SNP phylogeny of four F. tularensis whole genome sequences from the B.Br.013 group. The Georgian strain is highlighted in gray and is basal to the other three genomes. Newly identified branches (B.Br.027 and B.Br.026) are colored red and showed two major divisions within the B.Br.013 group. This phylogeny was rooted using OSU18 (not depicted). Bootstrap values are based on 1000 replicates in PAUP using a heuristic search.

\section{Identification of new lineages and subclades}

We designed assays targeting 21 of the 39 putative SNPs leading to the sequenced Georgian strain (Table 1) and screened them across the 25 Georgian isolates (Table 2) to reveal additional phylogenetic structure among these strains. All 21 SNPs were determined to be real and assigned the 25 strains to a monophyletic lineage (B. Br.027; also referred to below as the Georgian lineage) that includes six new subclades (Figure 2A). We also designed an assay (Table 1) targeting one of six putative SNPs along the branch (B.Br.026 in Figure 1B) leading to the other sequenced strains (FSC 200, LVS, and RC503) and screened it across DNA extracts from these three sequenced strains, as well as the 25 strains in the Georgian lineage. Consistent with the bioinformatics analyses, DNA extracts from the three sequenced strains all possessed the derived state for this SNP, whereas the 25 strains in the Georgian lineage all possessed the ancestral state for this SNP. This confirmed that the SNP was real and also branch B.Br.026, which leads to the lineage that gave rise to the previously known subclades within the B.Br.013 group [16]. Altogether, we identified a total of 7 new branches (B.Br.026-B.Br.032, Figure 2A) and designated a single canSNP for each of these branches with corresponding SNP genotyping assays (Table 1). Designating a single SNP as canonical 
Table 1 Melt-MAMA primers targeting informative canSNPs

\begin{tabular}{|c|c|c|c|c|c|c|c|}
\hline SNP & $\begin{array}{c}\text { SCHU } \\
\text { S4 } \\
\text { position }\end{array}$ & $\begin{array}{l}\text { Genome } \\
\text { SNP state } \\
(D / A)^{a}\end{array}$ & $\begin{array}{c}\text { Melt } \\
\text { MAMA } \\
\text { primer }^{c}\end{array}$ & Melt-MAMA primer sequences $^{d}$ & $\begin{array}{l}\text { Primer } \\
\text { conc. } \\
(\mu \mathrm{M})\end{array}$ & $\begin{array}{l}\text { Annealing } \\
\text { temp. }\left({ }^{\circ} \mathrm{C}\right)\end{array}$ & $\begin{array}{l}\text { Melting } \\
\mathrm{T}_{\mathrm{m}}\left({ }^{\circ} \mathrm{C}\right)\end{array}$ \\
\hline \multirow{3}{*}{$\begin{array}{c}\text { B. } \\
\text { Br.026 }\end{array}$} & 1484645 & $\mathrm{~A} / \mathrm{C}$ & $\mathrm{D}$ & GAAACTTATTTGTTCCTAAGACAGTGACACTA & 0.800 & 55 & 73.1 \\
\hline & & & A & ggggcggggcggggcAAACTTATTTGTTCCTAAGACAGTGACAgTC & 0.200 & & 79.7 \\
\hline & & & C & GCATTGAGTTTGACAGGGTTGC & 0.200 & & \\
\hline \multirow{3}{*}{$\begin{array}{c}\text { B. } \\
\text { Br.027 }\end{array}$} & 1329722 & $T / G^{b}$ & $\mathrm{D}$ & ggggcggggcggggcggggcCATGCCAGGCACTACAATTGATAGTaTA & 0.200 & 55 & 78.2 \\
\hline & & & A & TGCCAGGCACTACAATTGATAGTtTC & 1.000 & & 73.6 \\
\hline & & & C & TATACTTCTGACCATGGCGTTCAAAT & 0.200 & & \\
\hline \multirow{3}{*}{$\begin{array}{c}\text { B. } \\
\text { Br.028 }\end{array}$} & 212729 & $\mathrm{~T} / \mathrm{G}$ & $\mathrm{D}$ & ggggcggggcggggcggggCAAATTAGTTCAAATGTTAAATTTGATCCT & 0.200 & 55 & 75.8 \\
\hline & & & A & AAATTAGTTCAAATGTTAAATTTGATaCG & 0.200 & & 67.7 \\
\hline & & & C & CAAAATAAATCCCGTTGAGAATAGAA & 0.200 & & \\
\hline \multirow{3}{*}{$\begin{array}{c}\text { B. } \\
\text { Br.029 }\end{array}$} & 1185519 & $A / G$ & D & ggggcggggcggggcggggсTGCTTAATCTCATTGACTAGCTGTGgTA & 0.200 & 55 & 78 \\
\hline & & & A & TGCTTAATCTCATTGACTAGCTGTGaTG & 1.000 & & 70 \\
\hline & & & C & ACAAAGTTGAAACTATCGAGCATAAATC & 0.200 & & \\
\hline \multirow{3}{*}{$\begin{array}{c}\text { B. } \\
\text { Br.030 }\end{array}$} & 928335 & $\mathrm{~T} / \mathrm{G}$ & $\mathrm{D}$ & ggggcggggcggggcggggcTGTTGGGTCAAAGAGAGAAGTgTT & 0.200 & 55 & 78.2 \\
\hline & & & A & ATTGTTGGGTCAAAGAGAGAAGTaTG & 0.200 & & 70 \\
\hline & & & C & GCCACCAAAGAATACAGAGTAGTCAT & 0.200 & & \\
\hline \multirow{3}{*}{$\begin{array}{c}\text { B. } \\
\text { Br.031 }\end{array}$} & 1634565 & $A / G$ & D & ggggcggggcggggcggggcGCACCAATCGTATCTAATTGATCCA & 0.400 & 55 & 79 \\
\hline & & & A & GCACCAATCGTATCTAATTGATECG & 0.200 & & 70 \\
\hline & & & C & AACTTTGCTAAAACAAATGCTGTTGC & 0.200 & & \\
\hline \multirow{3}{*}{$\begin{array}{c}\text { B. } \\
\text { Br.032 }\end{array}$} & 283540 & $A / G^{b}$ & $\mathrm{D}$ & ggggcggggcggggcggggCTGCTAAACCTACAGTAATCAGAAGTATtAT & 0.200 & 55 & 72 \\
\hline & & & A & TGCTAAACCTACAGTAATCAGAAGTATCAC & 0.600 & & 68.4 \\
\hline & & & C & GCTAAATTTTAGTAAGATAAAAAGTGTAAGTAGTG & 0.200 & & \\
\hline
\end{tabular}

${ }^{a}$ SNP states are presented according to their orientation in the SCHU S4 reference genome (NC_006570);

${ }^{b}$ Assays designed from the reverse complement of the reference sequence.

${ }^{C} D$ : Derived; A: Ancestral; C: Common Primer

${ }^{d}$ Primer tails and antepenultimate mismatch bases are in lower case

for each branch maximizes phylogenetic information while minimizing the number of required assays by eliminating redundant SNPs, thus providing a highly efficient means of determining the phylogenetic positions of isolates for highly clonal pathogens such as $F$. tularensis [15,24]. In addition, canSNPs represent standardized phylogenetic positions for comparison in future studies performed by different research groups.

To understand the relationship of the Georgian lineage to other Eastern European lineages, we genotyped 132 geographically diverse group B.Br.013 isolates collected in Central and Eastern Europe across the B. Br.026 and B.Br.027 canSNP assays (Figure 2A, see additional file 3). All resulting genotypes from this analysis were phylogenetically consistent with no observed homoplasy. With just two exceptions, all of these isolates were assigned to the B.Br.026 lineage. The exceptions were two isolates from the Crimean region of Ukraine that were assigned to the Georgian lineage. Subsequent, additional canSNP analyses assigned these two isolates to the basal B.Br.027/028 subclade within the Georgian lineage. These results indicate that the Georgian isolates, as well as the two isolates from Crimea, are phylogenetically distinct from the previously described $F$. tularensis subsp. holarctica subpopulations.

The subclades within the Georgian lineage did not display a differentiated phylogeographic pattern but, rather, were spatially dispersed in a mixed fashion throughout Eastern Georgia and the Crimean region of Ukraine (Figure 2B). The assignment of the Crimean isolates to the basal B.Br.027/028 subclade within the Georgian lineage (Figure 2A) confirms that this lineage is not geographically restricted to Georgia, and is suggestive of a north to south dispersal pattern. That said, the overall geographic extent of the Georgian lineage is currently 
unknown due to the limited sampling in adjacent countries.

\section{Further discrimination using MLVA}

MLVA was used to examine genetic variation within each identified subclade of the Georgian lineage (Table 2; Additional file 4). Five unique MLVA genotypes were identified among the 25 Georgian isolates (Table 2) that were distinct from the MLVA genotypes of strains found north of Georgia. Calculations of MLVA diversity ( $D=G / N$ ) within each subclade (see methods for calculation) showed decreasing levels of diversity within higher resolution subclades (Figure 2A). The most basal Georgian subclade, B.Br.027/028 ( $\mathrm{D}=0.67$ ) (Figure 2A), was comprised of a single Georgian isolate that was distinguishable from the two Crimean isolates in the same subclade due to a distinct MLVA genotype. There were three MLVA genotypes among the seven Georgian isolates within subclade B.Br.028/029 ( $\mathrm{D}=0.43$ ). A single MLVA genotype was shared by all seven Georgian isolates in subclade B.Br.029/030 ( $\mathrm{D}=0.14)$, and the two other intermediate subclades (B.Br.030/031 and B.
Br.031/032) contained only a single isolate each. Only a single MLVA genotype was observed among these two isolates and the eight isolates in the terminal subclade B.Br.Georgia ( $\mathrm{D}=0.13$ in subclade B.Br.Georgia) (Figure 2A, Table 2). In general, MLVA diversity trended towards lower values nearer to the branch tip, consistent with shorter evolutionary times to generate diversity.

\section{Discussion}

The low number of SNPs found globally among $F$. tularensis subsp. holarctica isolates suggests that this subspecies only recently emerged through a genetic bottleneck and then rapidly dispersed across the Northern Hemisphere $[3,7,8,29,30]$. The phylogeographic model of Vogler et al. [15] suggests a North American derivation for the main $F$. tularensis subsp. holarctica radiation that spread throughout the Northern Hemisphere. However, previous analyses of the spread throughout Europe and Asia were hindered by a lack of isolates from the regions along the European/Asian juncture and in East Asia. This study begins to address this knowledge gap by describing additional phylogenetic structure based

Table 2 Francisella tularensis subsp. holarctica isolates from the country of Georgia used in this study

\begin{tabular}{|c|c|c|c|c|c|c|c|}
\hline $\mathrm{ID}^{a}$ & State/Province & County/Region & Location $^{b}$ & Source & Date & SNP Subclade ${ }^{c}$ & MLVA Genotype $^{d}$ \\
\hline F0677 & Shida Kartli & Gori & village Lamiskana & Haemaphysalis otophila & $03 / 00 / 2008$ & B.Br.027/028 & A \\
\hline F0658 & Shida Kartli & Kaspi & village Rene & water & $00 / 00 / 2007$ & B.Br.028/029 & B \\
\hline F0660 & Shida Kartli & Gori & village Nadarbazevi & Dermacentor marginatus & $00 / 00 / 2004$ & B.Br.028/029 & C \\
\hline F0662 & Samtskhe-Javakheti & Akhaltsikhe & village Minadze & fleas & 00/00/1997 & B.Br.028/029 & B \\
\hline F0674 & Shida Kartli & Kaspi & village Rene & Dermacentor marginatus & $04 / 00 / 2007$ & B.Br.028/029 & B \\
\hline F0675 & Shida Kartli & Gori & village Nadarbazevi & Haemaphysalis otophila & 04/00/2007 & B.Br.028/029 & B \\
\hline F0678 & Shida Kartli & Kaspi & village z/Rene & Dermacentor marginatus & 06/00/2008 & B.Br.028/029 & C \\
\hline F0679 & Shida Kartli & Kaspi & village z/Rene & Haemaphysalis sulcata & 06/00/2008 & B.Br.028/029 & $\mathrm{D}$ \\
\hline F0659 & Kvemo Kartli & Dmanisi & unknown & Microtus arvalis Pall. & $00 / 00 / 1990$ & B.Br.029/030 & A \\
\hline F0665 & Shida Kartli & Gori & village Shavshvebi & Gamasidae ticks & 00/00/1982 & B.Br.029/030 & A \\
\hline F0666 & Samtskhe-Javakheti & Aspindza & village Indusa & Dermacentor marginatus & $00 / 00 / 2004$ & B.Br.029/030 & A \\
\hline F0667 & Shida Kartli & Gori & village Nadarbazevi & Dermacentor marginatus & $00 / 00 / 2004$ & B.Br.029/030 & A \\
\hline F0668 & Shida Kartli & Gori & village Nadarbazevi & Dermacentor marginatus & $00 / 00 / 2004$ & B.Br.029/030 & A \\
\hline F0669 & Samtskhe-Javakheti & Ninotsminda & unknown & Dermacentor marginatus & $00 / 00 / 2002$ & B.Br.029/030 & A \\
\hline F0670 & Shida Kartli & Gori & village Tkviavi & Dermacentor marginatus & $00 / 00 / 2004$ & B.Br.029/030 & A \\
\hline F0672 & Shida Kartli & Gori & village Khurvaleti & Dermacentor marginatus & $00 / 00 / 2004$ & B.Br.030/031 & $\mathrm{E}$ \\
\hline F0655 & Kakheti & Dedoplis Tskaro & Solukh steppe & Meriones erythrurus Gray & 00/00/1956 & B.Br.031/032 & $\mathrm{E}$ \\
\hline F0656 & Kakheti & Dedoplis Tskaro & Nazarlebi Mountain & Ixodidae tick & 00/00/1956 & B.Br.Georgia & $\mathrm{E}$ \\
\hline F0657 & Shida Kartli & Tskhinvali & village Khetagurov & Sorex sp. & $00 / 00 / 1974$ & B.Br.Georgia & $\mathrm{E}$ \\
\hline F0661 & Samtskhe-Javakheti & Akhaltsikhe & village Klde & Microtus socialis Pall. & 00/00/1992 & B.Br.Georgia & $\mathrm{E}$ \\
\hline F0663 & Shida Kartli & Kareli & village Ruisi & Ixodidae tick & 00/00/1997 & B.Br.Georgia & $\mathrm{E}$ \\
\hline F0664 & Shida Kartli & Kareli & village Ruisi & wheat & 00/00/1997 & B.Br.Georgia & $\mathrm{E}$ \\
\hline F0671 & unknown & unknown & East Georgia & unknown & unknown & B.Br.Georgia & $\mathrm{E}$ \\
\hline F0673 & unknown & unknown & East Georgia & unknown & unknown & B.Br.Georgia & $\mathrm{E}$ \\
\hline F0676 & Shida Kartli & Gori & village Nadarbazevi & Dermacentor marginatus & $05 / 00 / 2007$ & B.Br.Georgia & $\mathrm{E}$ \\
\hline
\end{tabular}

${ }^{a}$ Strain ID in the Northern Arizona University DNA collection

${ }^{b}$ City, Town, or Village

${ }^{c}$ canSNP lineage

${ }^{d}$ Genotypes (A to E) determined by MLVA11 system (Vogler et al, 2009). 


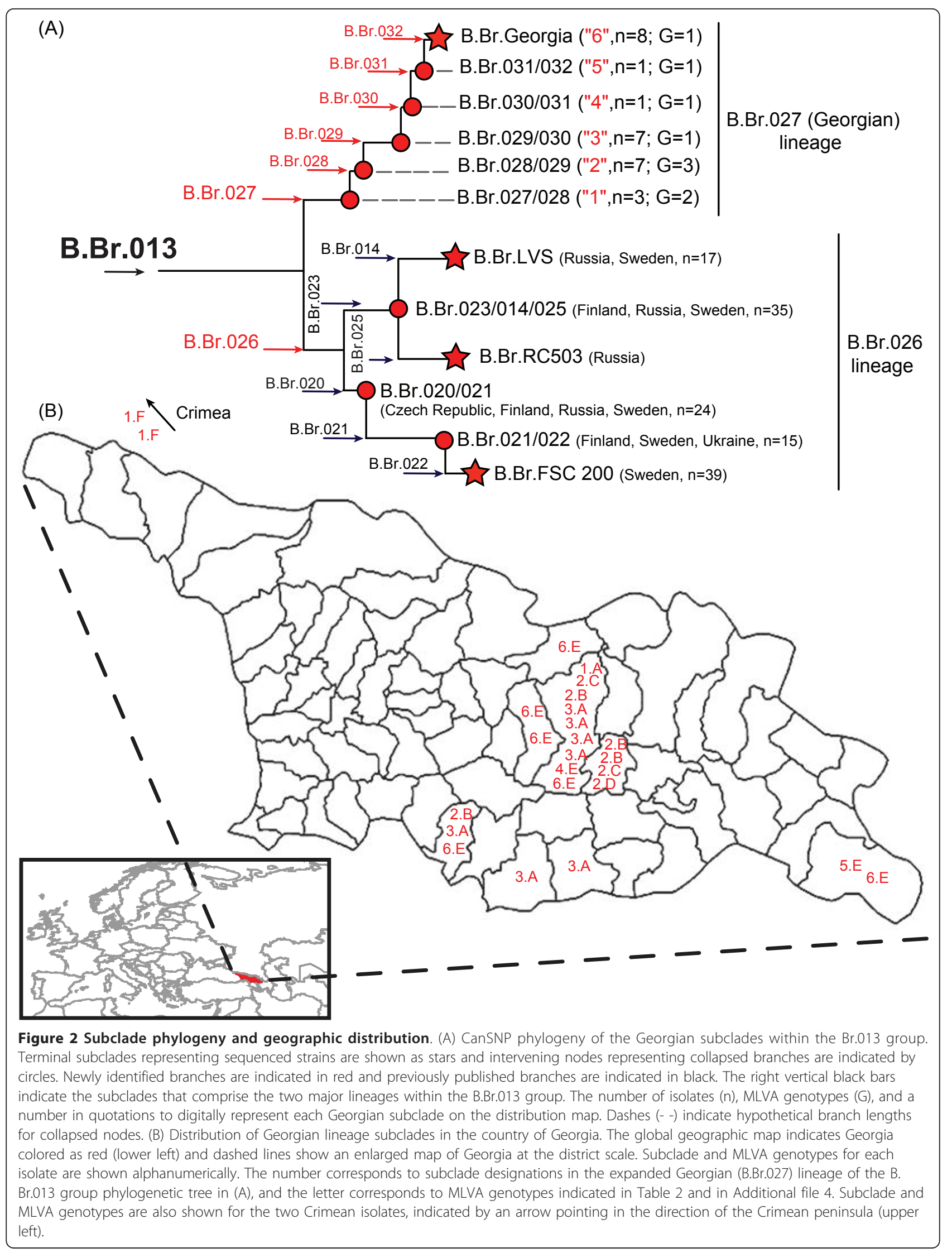


upon 25 isolates from the European/Asian border country of Georgia through the use of SNPs discovered from whole genome comparisons.

Whole genome sequencing of a Georgian strain revealed SNPs that placed the Georgian lineage basal to the diversification of the subclades of the B.Br.026 lineage within the B.Br.013 group $[15,16]$ (Figure 1B). In addition, a relatively large number of subclades (phylogenetic topology) within the Georgian lineage were discovered amongst a relatively small number of Georgian isolates. This is fortuitous, and perhaps a consequence of the selection of Georgian strain F0673 for sequencing [31,32].

Georgian (B.Br.027) lineage isolates are geographically distinct from the B.Br.026 lineage isolates. Georgian lineage isolates appear restricted to regions of the Ukraine and Georgia, whereas the B.Br.026 lineage isolates are concentrated in Central-Eastern Europe, based upon the isolates examined here. However, the true geographic extent of the Georgian lineage could not be fully determined due to the lack of a comprehensive set of isolates from regions neighboring Georgia. That said, it is clear that the Georgian lineage is absent from Central Europe. The geographic division of the B.Br.013 and B. Br.FTNF002-00 groups into Eastern and Western Europe, respectively, suggests that the common ancestor to these two lineages, and possibly the Georgian and north of Georgia lineages (B.Br.027 and B.Br.026, respectively), existed west of Georgia, although the lack of a comprehensive set of Asian isolates limits our ability to draw conclusions about the F. tularensis subsp. holarctica radiation that spread throughout Eurasia. Likewise, data from our current collection of isolates suggest that $F$. tularensis was introduced into Georgia from the north, though we unfortunately lack comparable isolates from the Middle East. For the entire F. tularensis subsp. holarctica radiation in Eurasia, a Scandinavian origin remains the most robust hypothesis given that Sweden contains the most phylogenetically diverse set of isolates in Eurasia, including isolates found in the subclade immediately basal to the B.Br.013 group [15].

Interestingly, at this regional scale, canSNPs and MLVA exhibited considerable congruence in identifying genetic groups. Specifically, canSNPs identified six subclades and MLVA identified five, albeit with slightly different but not phylogenetically inconsistent membership due to the nature of the two different marker types. SNPs discovered from whole genome sequences will typically provide greater discrimination than MLVA, as seen in subclades B.Br.030/031, B.Br.031/032 and B.Br. Georgia (Table 2), and can even be used to identify specific strains [33]. However, discovering these rare SNPs requires whole genome sequencing whereas MLVA can identify nearly the same number of genetic groups by simply surveying a few highly polymorphic portions of the genome. At this regional scale, homoplasy does not appear to be much of a factor in obscuring phylogenetic signal for identifying genetic groups using MLVA, although the relationships among those groups are less resolved as isolates from adjacent groups share MLVA genotypes. Together, SNPs and MLVA provide complementary approaches, by first accurately placing isolates in a phylogeny using SNPs and then discriminating among isolates within SNP-determined subclades using MLVA. This step-wise approach has been termed Progressive Hierarchical Resolving Assays using Nucleic Acids (PHRANA) [24].

\section{Conclusions}

We describe a new subpopulation in the B.Br.013 group from Georgia that is genetically and geographically distinct from the other B.Br.013 group subpopulations found in Europe. Members of this new lineage are endemic to parts of Eastern Europe and Western Asia, though the complete geographic range remains unknown. The basal positioning of the Georgian lineage and its restricted geographic distribution illustrates the need for studies on additional Asian and East European isolates to gain a better understanding of the clonal expansion of $F$. tularensis subsp. holarctica.

\section{Methods}

\section{Whole Genome Sequencing}

We sequenced a single Georgian isolate (F0673), representing the most common MLVA profile type of $F$. tularensis subsp. holarctica found in the country of Georgia (Chanturia, unpubl. data), using Illumina's Genome Analyzer II (San Diego, CA). DNA from F0673 was prepared using a standard chloroform extraction protocol [34]. Library preparation for this isolate involved sonication of $5 \mu \mathrm{g}$ genomic DNA to an average fragment size of $350 \mathrm{bp}$, followed by sample preparation and cluster generation protocols for paired-end reads from Illumina. The library was quantified using SYBR-based qPCR and primers modified from the adaptor sequence. The library was then run in two lanes of the flow cell to increase overall coverage. Read lengths were ca. $40 \mathrm{bp}$, with a final yield of $32 \mathrm{~Gb}$ of sequence for the entire run. Image analysis for base calling and alignments followed the methods of Craig and colleagues [35]. The entire Sequence Read Archive of F0673 was deposited to GenBank (SRP003002.2).

\section{SNP Discovery and Analysis}

To identify putative SNPs, the Georgian isolate WGS was aligned with LVS ( $F$. tularensis subsp. holarctica LVS NC_007880) and was compared to four other WGSs available from GenBank (F. tularensis subsp. 
holarctica FSC 200 NZ_AASP00000000, F. tularensis subsp. holarctica LVS NC_007880 and F. tularensis subsp. holarctica OSU18 NC_008369) and the Human Genome Sequencing Center at Baylor College of Medicine (F. tularensis subsp. holarctica RC503 http://www. hgsc.bcm.tmc.edu/microbial-detail.xsp?project_id=144). Three of these WGSs (FSC 200, LVS, and RC503) were selected because of their membership in the B.Br.013 group, whereas the OSU18 WGS was selected as an outgroup. F. tularensis subsp. tularensis SCHU S4 (NC_006570) was used for referencing SNP positions. Two independent approaches were used for SNP discovery, the MAQ algorithm [36] and a custom SNP calling pipeline. The in-house pipeline used for SNP discovery first compares WGSs in a pairwise fashion using MUMmer [37] to identify putative SNPs and then uses PERL and Java Scripts for grouping the discovered SNPs by shared location, comparing SNPs across all taxa and tabulating the final putative SNP set according to certain criteria. Specifically, SNPs from repeated regions, including paralogous genes, apparent tri-state SNPs and SNPs with an adjacent SNP closer than $11 \mathrm{bp}$ away were removed from analysis. Furthermore, the SNP locus must be present in all of the genomes to be included in the analysis. The software package PAUP 4.0b10 (D. Swofford, Sinauer Associates, Inc., Sunderland, MA) was used to construct a whole genome SNP phylogeny (Figure $1 \mathrm{~B})$ using maximum parsimony.

\section{CanSNP Selection and Analysis}

Thirty-nine putative SNPs specific to the Georgian lineage were identified in the whole genome sequence analysis. Of these, twenty-one were incorporated into meltMAMA genotyping assays, as previously described [15], except that only GC- rich tails were used on one allele specific primer [38]. A melt-MAMA assay was also designed for branch B.Br.026 within the B.Br.013 group. Allele-specific melt-MAMA primers were designed using Primer Express 3.0 software (Applied Biosystems, Foster City, CA) (Table 1). All other assay reagents and instrumentation were as previously described [15]. DNA templates were extracted using either chloroform [34] or DNeasy blood and tissue kits (Qiagen, Valencia, CA). Reactions were first raised to $50^{\circ} \mathrm{C}$ for 2 min to activate the uracil glycolase, then raised to $95^{\circ} \mathrm{C}$ for $10 \mathrm{~min}$ to denature the DNA and then cycled at $95^{\circ} \mathrm{C}$ for $15 \mathrm{~s}$ and $55^{\circ} \mathrm{C}$ for $1 \mathrm{~min}$ for 33 cycles (Table 1 ). Immediately after the completion of the PCR cycle, amplicon melt dissociation was measured by ramping from $60^{\circ} \mathrm{C}$ to $95^{\circ}$ $\mathrm{C}$ in $0.2^{\circ} \mathrm{C} / \mathrm{min}$ increments and recording the fluorescent intensity. The genome locations, primer sequences and annealing temperatures for the seven canSNP assays can be found in Table 1. We screened a geographically diverse panel of 132 European isolates belonging to the
B.Br.013 group and a geographically diverse panel of 25 Georgian isolates across lineage-specific assays to determine whether they were in the B.Br.026 or the Georgian (B.Br.027) lineages (see additional file 3, Table 2).

\section{MLVA}

All 25 Georgian isolates were screened with an 11-marker MLVA system (Additional file 4) [25]. This was done in order to determine the level of genetic diversity within each identified subclade. The MLVA Diversity (D) was calculated for each subclade using the following equation: $\mathrm{G} / \mathrm{N}$ ( $\mathrm{G}$ = MLVA genotypes; $\mathrm{N}=$ number of isolates). Diversity was not calculated for subclades with a single isolate.

\section{Additional material}

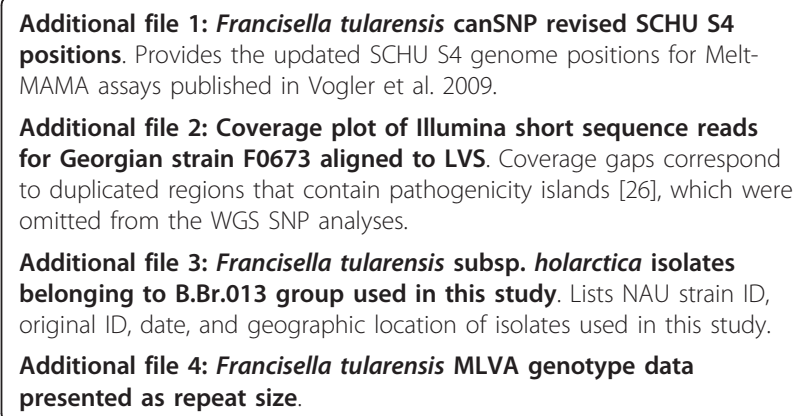

Additional file 3: Francisella tularensis subsp. holarctica isolates belonging to B.Br.013 group used in this study. Lists NAU strain ID, original ID, date, and geographic location of isolates used in this study.

Additional file 4: Francisella tularensis MLVA genotype data presented as repeat size.

\section{Acknowledgements}

This work was funded by the U.S. Department of Homeland Security S\&T CB Division Bioforensics R\&D Program. Note that the use of products/names does not constitute endorsement by the Department of Homeland Security of the United States.

\section{Author details}

${ }^{1}$ Center for Microbial Genetics and Genomics, Northern Arizona University, Flagstaff, AZ 86011-4073, USA. ²National Center for Disease Control and Public Health, Tbilisi, 0177, Georgia. ${ }^{3}$ Translational Genomics Research Institute, Phoenix, AZ 85004, USA. ${ }^{4}$ Veterinary Medical Research Institute, Hungarian Academy of Sciences, Budapest, Hungary. ${ }^{5}$ US Army Medical Research Institute of Infectious Diseases, Fort Detrick, Frederick, Maryland 21702-5011, USA.

\section{Authors' contributions}

GC and DNB carried out the molecular genetic studies, constructed the figures, performed data analysis, and drafted the manuscript. EZ and GB carried out the molecular genetic studies, MK, NT, ST, PI, JF assisted in the design of the study. SMBS, JSBS, SS, and MDC participated in the computational in silico data analyses. JTF sequenced the Georgian strain. MG, AHP, and ELK carried out the molecular genetic studies. AJV participated in the design of the study and drafted the manuscript. JDB and TP drafted the manuscript. DMW assisted in the design of the study and drafted the manuscript. PK participated in the project design, data interpretation and drafted the manuscript. All authors read and approved of the final manuscript.

\section{Authors' information}

GC, MS, National Center for Disease Control and Public Health, Tbilisi, Georgia

DNB, PhD, Northern Arizona University, Flagstaff, Arizona 
MK, PhD, National Center for Disease Control and Public Health, Tbilisi, Georgia

EZ, MS, National Center for Disease Control and Public Health, Tbilisi, Georgia

GB, MS, National Center for Disease Control and Public Health, Tbilisi, Georgia

NT, MD, Ph.D., National Center for Disease Control and Public Health, Tbilisi, Georgia

ST, MD, Ph.D., National Center for Disease Control and Public Health, Tbilisi, Georgia

Pl, MD, Ph.D., National Center for Disease Control and Public Health, Tbilisi, Georgia

JF, Ph.D., U.S. Army Medical Research Institute of Infectious Diseases, Fort Detrick, Frederick, Maryland

SMBS, PhD, Translational Genomics Research Institute, Phoenix, Arizona

JSBS, BS, Translational Genomics Research Institute, Phoenix, Arizona

SS, MS, Translational Genomics Research Institute, Phoenix, Arizona

MDC, PhD, Translational Genomics Research Institute, Flagstaff, Arizona

MG, DVM, MSc, Veterinary Medical Research Institute, Hungarian Academy of Sciences, Budapest, Hungary

AHP, Northern Arizona University, Flagstaff, Arizona

ELK, Northern Arizona University, Flagstaff, Arizona

JDB, PhD, Northern Arizona University, Flagstaff, Arizona

TP, PhD, Northern Arizona University, Flagstaff, Arizona

JTF, PhD, Northern Arizona University, Flagstaff, Arizona

AJV, PhD, Northern Arizona University, Flagstaff, Arizona

DMW, PhD, Northern Arizona University, Flagstaff, Arizona

PK, PhD, Northern Arizona University, and Translational Genomics Research Institute, Flagstaff, Arizona

Received: 4 March 2011 Accepted: 17 June 2011

Published: 17 June 2011

\section{References}

1. Dennis DT, Inglesby TV, Henderson DA: Tularemia as a biological weapon: medical and public health management. Working group on Civilian Biodefense. JAMA 2001, 285:2763-2773, 15 other authors,

2. Huber BE, Escudero R, Busse HJ, Seibold E, Scholz HC, Anda P, Kampfer P, Splettstoesser WD: Description of Francisella hispaniensis sp. nov., isolated from human blood, reclassification of Francisella novicida (Larson et al. 1955) Olsufiev et al. 1959 as Francisella tularensis subsp. novicida comb. nov., and emended description of the genus Francisella. Int J Syst Evol Microbiol 2009

3. Keim P, Johansson A, Wagner DM: Molecular epidemiology, evolution, and ecology of Francisella. Ann N Y Acad Sci 2007, 1105:30-66.

4. Johansson A, Celli J, Conlan W, Elkins KL, Forsman M, Keim PS, Larsson P, Manoil C, Nano FE, Petersen JM, Sjostedt A: Objections to the transfer of Francisella novicida to the subspecies rank of Francisella tularensis. Int J Syst Evol Microbiol 2010, 60:1717-1718, author reply 1718-1720.

5. Staples JE, Kubota KA, Chalcraft LG, Mead PS, Petersen JM: Epidemiologic and molecular analysis of human tularemia, United States, 1964-2004. Emerg Infect Dis 2006, 12:1113-1118.

6. Svensson $K$, Larsson $P$, Johansson D, Byström $M$, Forsman $M$, Johansson $A$ : Evolution of subspecies of Francisella tularensis. J Bacteriol 2005, 187:3903-3908

7. Johansson A, Farlow J, Larsson P, Dukerich M, Chambers E, Byström M, Fox J, Chu M, Forsman M, Sjöstedt A, Keim P: Worldwide genetic relationships among Francisella tularensis isolates determined by multiple-locus variable-number tandem repeat analysis. J Bacterio/ 2004 186:5808-5818

8. Farlow J, Wagner DM, Dukerich M, Stanley M, Chu M, Kubota K, Petersen J, Keim P: Francisella tularensis in the United States. Emerg Infect Dis 2005, 11:1835-1841.

9. Petersen JM, Molins CR: Subpopulations of Francisella tularensis ssp. tularensis and holarctica: identification and associated epidemiology. Future Microbiol 2010, 5:649-661.

10. Gurcan S, Karabay O, Karadenizli A, Karagol C, Kantardjiev T, Ivanov IN: Characteristics of the Turkish isolates of Francisella tularensis. Jpn J Infect Dis 2008, 61:223-225.

11. Chitadze N, Kuchuloria T, Clark DV, Tsertsvadze E, Chokheli M, Tsertsvadze N, Trapaidze N, Lane A, Bakanidze L, Tsanava S, Hepburn MJ,
Imnadze P: Water-borne outbreak of oropharyngeal and glandular tularemia in Georgia: investigation and follow-up. Infection 2009, 37:514-521.

12. Akalin $\mathrm{H}$, Helvaci $\mathrm{S}$, Gedikoglu S: Re-emergence of tularemia in Turkey. Int J Infect Dis 2009, 13:547-551.

13. Whipp MJ, Davis JM, Lum G, de Boer J, Zhou Y, Bearden SW, Petersen JM, Chu MC, Hogg G: Characterization of a novicida-like subspecies of Francisella tularensis isolated in Australia. J Med Microbiol 2003, 52:839-842.

14. Birdsell DN, Stewart T, Vogler AJ, Lawaczeck E, Diggs A, Sylvester $T L$, Buchhagen JL, Auerbach RK, Keim P, Wagner DM: Francisella tularensis subsp. novicida isolated from a human in Arizona. BMC Res Notes 2009, 2:223.

15. Vogler AJ, Birdsell D, Price LB, Bowers JR, Beckstrom-Sternberg SM, Auerbach RK, Beckstrom-Sternberg JS, Johansson A, Clare A, Buchhagen JL, Petersen JM, Pearson T, Vaissaire J, Dempsey MP, Foxall P, Engelthaler DM, Wagner DM, Keim P: Phylogeography of Francisella tularensis: global expansion of a highly fit clone. J Bacteriol 2009, 191:2474-2484.

16. Svensson K, Granberg M, Karlsson L, Neubauerova V, Forsman M, Johansson A: A real-time PCR array for hierarchical identification of Francisella isolates. PLoS One 2009, 4:e8360.

17. Pilo P, Johansson A, Frey J: Identification of Francisella tularensis cluster in central and western Europe. Emerg Infect Dis 2009, 15:2049-2051.

18. Vogler AJ, Birdsell DN, Lee J, Vaissaire J, Doujet CL, Lapalus M, Wagner DM, Keim P: Phylogeography of Francisella tularensis ssp. holarctica in France. Letters in Applied Microbiology 2010, 52:177-180.

19. Johansson A, Berglund L, Eriksson U, Göransson I, Wollin R, Forsman M, Tärnvik A, Sjöstedt A: Comparative analysis of PCR versus culture for diagnosis of ulceroglandular tularemia. J Clin Microbiol 2000, 38:22-26.

20. Egorova LS, I'in VA, Algazin IP, Mal'kov GB: [Isolation of the causative agent of tularemia from Siberian lemmings in Eastern Taymyr]. Zh Mikrobiol Epidemiol Immunobiol 1975, 128-132.

21. Zhang F, Liu W, Chu MC, He J, Duan Q, Wu XM, Zhang PH, Zhao QM, Yang H, Xin ZT, Cao WC: Francisella tularensis in rodents, China. Emerg Infect Dis 2006, 12:994-996.

22. Vodop'ianov AS, Mishan'kin BN, Pavlovich NV, Pichurina NL: [Genotypic heterogeneity and geographic diversity of collection strains of Francisella tularensis as determined using the VNTR variability analysis and DNA sequencing]. Mol Gen Mikrobiol Virusol 2007, 33-40.

23. Zhang F, Liu W, Wu XM, Xin ZT, Zhao QM, Yang H, Cao WC: Detection of Francisella tularensis in ticks and identification of their genotypes using multiple-locus variable-number tandem repeat analysis. BMC Microbiol 2008, 8:152.

24. Keim P, Van Ert MN, Pearson T, Vogler AJ, Huynh LY, Wagner DM: Anthrax molecular epidemiology and forensics: using the appropriate marker for different evolutionary scales. Infect Genet Evol 2004, 4:205-213.

25. Vogler AJ, Birdsell D, Wagner DM, Keim P: An optimized, multiplexed multi-locus variable-number tandem repeat analysis system for genotyping Francisella tularensis. Lett Appl Microbiol 2009, 48:140-144

26. Bröms JE, Sjöstedt A, Lavander M: The role of the Francisella tularensis pathogenicity island in type $\mathrm{VI}$ secretion, intracellular survival, and modulation of host cell signaling. Frontiers in Microbiology 2010, 1:1-17.

27. Larsson $P$, Elfsmark $D$, Svensson $K$, Wikstrom $P$, Forsman $M$, Brettin $T$, Keim $P$, Johansson A: Molecular evolutionary consequences of niche restriction in Francisella tularensis, a facultative intracellular pathogen. PLoS Pathog 2009, 5:e1000472.

28. Broekhuijsen M, Larsson P, Johansson A, Bystrom M, Eriksson U, Larsson E, Prior RG, Sjostedt A, Titball RW, Forsman M: Genome-wide DNA microarray analysis of Francisella tularensis strains demonstrates extensive genetic conservation within the species but identifies regions that are unique to the highly virulent F. tularensis subsp. tularensis. J Clin Microbiol 2003, 41:2924-2931.

29. Dempsey MP, Nietfeldt J, Ravel J, Hinrichs S, Crawford R, Benson AK: Pairedend sequence mapping detects extensive genomic rearrangement and translocation during divergence of Francisella tularensis subsp. tularensis and Francisella tularensis subsp. holarctica populations. J Bacteriol 2006, 188:5904-5914

30. Larsson P, Svensson K, Karlsson L, Guala D, Granberg M, Forsman M, Johansson A: Canonical insertion-deletion markers for rapid DNA typing of Francisella tularensis. Emerg Infect Dis 2007, 13:1725-1732. 
31. Pearson T, Busch JD, Ravel J, Read TD, Rhoton SD, U'Ren JM, Simonson TS, Kachur SM, Leadem RR, Cardon ML, Van Ert MN, Huynh LY, Fraser CM Keim P: Phylogenetic discovery bias in Bacillus anthracis using singlenucleotide polymorphisms from whole-genome sequencing. Proc Natl Acad Sci USA 2004, 101:13536-13541.

32. Pearson T, Okinaka RT, Foster JT, Keim P: Phylogenetic understanding of clonal populations in an era of whole genome sequencing. Infect Genet Evol 2009, 9:1010-1019.

33. Vogler AJ, Driebe EM, Lee J, Auerbach RK, Allender CJ, Stanley M, Kubota K, Andersen GL, Radnedge L, Worsham PL, Keim P, Wagner DM: Assays for the rapid and specific identification of North American Yersinia pestis and the common laboratory strain CO92. Biotechniques 2008, 44:201, 203204, 207.

34. Sambrook J, Fritsch EF, Maniatis T: Molecular Cloning: a Laboratory Manual. Cold Spring Harbor, NY: Cold Spring Harbor Laboratory; 21989.

35. Craig DW, Pearson JV, Szelinger S, Sekar A, Redman M, Corneveaux JJ, Pawlowski TL, Laub T, Nunn G, Stephan DA, Homer N, Huentelman MJ: Identification of genetic variants using bar-coded multiplexed sequencing. Nat Methods 2008, 5:887-893.

36. Li H, Ruan J, Durbin R: Mapping short DNA sequencing reads and calling variants using mapping quality scores. Genome Res 2008, 18:1851-1858.

37. Kurtz S, Phillippy A, Delcher AL, Smoot M, Shumway M, Antonescu C, Salzberg SL: Versatile and open software for comparing large genomes. Genome Biology 2004, 5:R12.

38. Papp AC, Pinsonneault JK, Cooke G, Sadee W: Single nucleotide polymorphism genotyping using allele-specific PCR and fluorescence melting curves. Biotechniques 2003, 34:1068-1072.

doi:10.1186/1471-2180-11-139

Cite this article as: Chanturia et al.: Phylogeography of Francisella tularensis subspecies holarctica from the country of Georgia. BMC Microbiology 2011 11:139.

\section{Submit your next manuscript to BioMed Central and take full advantage of:}

- Convenient online submission

- Thorough peer review

- No space constraints or color figure charges

- Immediate publication on acceptance

- Inclusion in PubMed, CAS, Scopus and Google Scholar

- Research which is freely available for redistribution

Submit your manuscript at www.biomedcentral.com/submit 